\title{
Sleep Inertia and Autonomic Effects on Post-Nap P300 Event-Related Potential
}

\author{
Masaya TAKAHASHI* and Heihachiro ARITO
}

\author{
National Institute of Industrial Health, 21-1, Nagao 6 chome, Tama-ku, Kawasaki 214-8585, Japan
}

Received June 8, 1998 and accepted August 10, 1998

\begin{abstract}
The objective of this study was to examine the relationship between post-nap measures of alertness and performance and non-rapid eye movement (NREM) sleep and parasympathetic activity during brief naps. Thirty healthy subjects were randomly assigned to no-nap, 15-min, and 45-min nap conditions after normal home sleep at prior night. Each nap was taken after lunch and monitored by electroencephalogram (EEG), electromyogram, electrooculogram, and electrocardiogram (ECG). Deep NREM sleep was quantified by EEG delta power density and the parasympathetic activity was quantified by the ECG high-frequency (HF) component of R-R interval variability during the 15- and 45-min naps. The P300 event-related potential, subjective sleepiness, and performance on a 90-min English transcription task were measured $30 \mathrm{~min}$ and $3 \mathrm{hr}$ after the naps and tested for their association with the EEG and ECG measures. A positive correlation was obtained between EEG delta power density during the naps and $P 300$ latency $30 \mathrm{~min}$ after the naps $(r=0.476, p<0.05)$. The HF component during the naps was negatively correlated with the P300 latency $3 \mathrm{hr}$ after the naps $(r=-0.519, p<0.05)$. These results suggest that the sleep inertia prolongs the $P 300$ latency immediately after the naps, and that the parasympathetic predominance during the naps may improve subsequent alertness as assessed by the shortened P300 latency $3 \mathrm{hr}$ after the naps.
\end{abstract}

Key words: Nap, Sleep inertia, Alertness, P300, Autonomic balance

\section{Introduction}

Accumulated evidence indicates that impaired alertness and performance can be counteracted by taking a nap ${ }^{1-6)}$. One of the potential disadvantages of napping, however, is sleep inertia: reductions in alertness and performance occurring immediately after being awakened from sleep ${ }^{7,8}$. Sleep inertia is a transient phenomenon, which is reported to dissipate within approximately 30 minutes. It has been hypothesized that sleep inertia is greatest when large amounts of deep non-rapid eye movement (NREM) occur during the $\operatorname{nap}^{7-11)}$.

In a previous study ${ }^{6}$, we observed that a 15-min daytime nap produced a decrease in the latency of P300 event-related potential (ERP) 30 min after awakening from the nap. A

\footnotetext{
*To whom correspondence should be addressed.
}

45-min daytime nap had little effect. The P300 ERP has been thought not only as a neurophysiological correlate of cognitive processes ${ }^{12)}$, but also as an objective marker of alertness ${ }^{13)}$. We assumed that this result could be explained by sleep inertia because the 45-min nap included deep NREM sleep as measured by visually scored slow-wave (stages $3+4$ ) sleep (SWS; mean $4.7 \mathrm{~min}$ ), while the 15 -min nap did not. A definite conclusion, however, can not be drawn before quantitatively assessing depth of the 15- and 45-min naps, and then examining the relationship between the depth of nap sleep and post-nap P300 latency. Toward this end, we applied a power spectrum analysis to the sleep electroencephalogram (EEG). This procedure was expected to provide more sensitive information about deep NREM sleep than conventional visual scoring techniques ${ }^{14-16)}$, especially during the 15-min nap where no SWS was apparent from visual scoring. 
Our previous study also showed that the parasympathetic activity increased during the 45 -min nap as revealed by an enhanced high-frequency (HF) component of electrocardiographic (ECG) R-R interval variability ${ }^{6}$. The HF component is reported to increase as the NREM sleep becomes deeper ${ }^{17},{ }^{18)}$, indicating parasympathetic predominance. Yet, effects of autonomic changes during sleep on subsequent alertness and performance are not determined. This may be due to the fact that past research examined sleep inertia exclusively in terms of EEG parameters during sleep ${ }^{9,19-21)}$. Based upon the results of our previous study, we hypothesized that the parasympathetic activity might be associated with post-nap change in P300 latency.

The purpose of the present study was to examine whether EEG delta power density and the HF component of the R-R interval variability during naps would be associated with the following post-nap parameters: the P300 measures, subjective sleepiness, and task performance. The time-course changes in alertness, performance, and autonomic balance with respect to three lengths of the naps $(0,15$, and $45 \mathrm{~min})$ have been reported previously ${ }^{6}$. The present study focused upon the post-nap parameters under the 15- and 45-min nap conditions in light of sleep inertia and autonomic balance during the naps.

\section{Methods}

\section{Subjects}

Thirty subjects with no history of sleep or cardiovascular disorders (11 males, 19 females, aged 20-30 years) were paid to participate in the experiment. All subjects gave informed consent, and the study protocol was approved by the Ethic Committee of our Institute.

\section{Procedure}

The experimental procedure has been described previously ${ }^{6}$. Briefly, the subjects practiced English transcription and $\mathrm{P} 300$ oddball tasks and were instructed to sleep at night for $7 \mathrm{hrs}$ while being monitored with a wrist activity monitor (Motionlogger Actigraph, Ambulatory Monitoring, USA) on the 1st day of the experiment. The actigraphic data confirmed that mean total sleep time was $7.4 \pm 1.0$ (SD) $h$. The subjects were randomly assigned to one of three nap conditions (0-, 15-, and 45-min time in bed; each $\mathrm{n}=10$ ) on the following day. Nap opportunities for 15 or 45 min were given after a lunch break on a bed in a darkened, electrically shielded chamber at 12:30. The 15- and 45-min naps were monitored polygraphically with the EEG, electrooculogram (EOG), electromyogram (EMG), and ECG. During the no-nap condition and a post-nap 30min period of casual reading on a chair outside the chamber, no polygraphic recordings were made. The P300 ERP, subjective sleepiness on Visual Analogue Scale ${ }^{22)}$ (VAS), and 5-min ECG were measured before (10:30), 30 min after (13:30/14:00 [45-min nap condition]), and $3 \mathrm{hr}$ after (16:30) the naps. These measurements were followed by a 90 -min English transcription task. The electrophysiological signals were recorded with an electroencephalograph (EEG-4217, Nihon Kohden, Japan) and were stored simultaneously on an FM tape recorder (XR-7000L, TEAC, Japan).

\section{Analysis of sleep EEG during naps}

The sleep EEG (Cz-A1+A2) signal was filtered (highfrequency cutoff: $30 \mathrm{~Hz}$ ) and digitized with a sampling rate of $125 \mathrm{~Hz}$. The EEG signal was then subjected to the power spectrum analysis by a fast Fourier transform (FFT) routine for consecutive 4-sec epochs which were averaged for each $20 \mathrm{sec}$ period (EEG Analyzer, Medical Research Equipment, Japan). A Hanning window was applied. The delta power density was calculated by integrating powers across 0.5 $4.0 \mathrm{~Hz}$. Epochs contaminated by artifacts were identified visually and excluded from the analysis. The resultant delta power density was further integrated over the entire NREM sleep episode during the naps to calculate slow-wave energy ${ }^{15)}$ (SWE) which was then log-transformed (logSWE). Sleep stages were scored visually in 20 -sec epochs according to the criteria of Rechtschaffen and Kales ${ }^{23}$.

P300 ERP, $R-R$ interval variability during naps, and Performance of English transcription task

The methods for recording and analysis of these measures have been reported in a previous paper ${ }^{6}$. Briefly, in the P300 oddball task, two hundred stimuli consisting of 1000 (non-target, 80\%) and 2000 (target, 20\%) Hz tones were randomly presented with an interstimulus interval of $2 \mathrm{sec}$ via a headphone at $50 \mathrm{~dB}$ sound pressure level. Subjects responded to each target tone by pressing a button as fast as possible with the thumb of their preferred hand. The EEG ( $\mathrm{Fz}, \mathrm{Cz}$, and $\mathrm{Pz}$ sites) and vertical EOG signals were digitized at $1000 \mathrm{~Hz}$ for $1100 \mathrm{msec}$ with a $100 \mathrm{msec}$ pre-stimulus baseline. Trials on which the EEG or EOG exceeded \pm 50 $\mu \mathrm{V}$ and those with erroneous responses were excluded from averaging. The $\mathrm{P} 300$ amplitude was measured at the $\mathrm{Pz}$ site as the largest positive peak occurring between $250-500$ msec post-stimulus, relative to the average of the 
corresponding $100 \mathrm{msec}$ pre-stimulus baseline. The P300 latency was measured at the $\mathrm{Pz}$ site as the time point of the largest $\mathrm{P} 300$ amplitude from the stimulus onset.

For the ECG analysis, the successive R-R interval spectra were calculated per 5 min period over the 15 - and 45 -min naps. The R-R intervals over 256 -sec epoch during each 5min segment, interpolated linearly, were subjected to FFT power spectrum analysis. The low-frequency (LF) and highfrequency (HF) powers were computed by integrating corresponding powers over $0.05-0.15 \mathrm{~Hz}$ and over $0.15-$ $0.5 \mathrm{~Hz}$, respectively. Percentage values (\%LF, \%HF) were calculated as the LF or HF power relative to the total power integrating over $0.02-0.50 \mathrm{~Hz}$. Mean R-R intervals (meanRR) also were obtained. Each R-R interval measure was obtained by averaging values during each 5 -min segment of the nap which contained less than $2.5 \mathrm{~min}$ of wakefulness. The total number of these segments was 16 and 60 for the 15- and 45-min naps, respectively.

As a performance measure, error rate in the English transcription task was calculated by dividing number of words transcribed erroneously by the total number of words transcribed during each 90 -min task period. These proportions were transformed to the arcsine of their square roots for statistical analysis. The total number of words transcribed during the 90-min period was not used in the correlation analysis since our previous study showed no significant difference in this measure between the nap conditions ${ }^{6}$.

\section{Statistical analysis}

Our primary interest was to determine the relationship between EEG and autonomic parameters during the naps and post-nap alertness and performance. Therefore, data obtained under the 15- $\min (n=9)$ and 45-min $(n=10)$ nap conditions were used for this analysis. Data from the nonap condition and one subject who was unable to nap during the 15-min nap was excluded from the analysis. Spearman rank order correlation coefficients were computed separately between the logSWE, \%HF, \%LF, meanRR during napping and the P300 latency and amplitude, subjective sleepiness, and English transcription error rates measured $30 \mathrm{~min}$ and $3 \mathrm{hr}$ after napping (SAS statistical package, Release 6.11, SAS Institute, USA).

\section{Results}

Figure 1 shows time courses of log-transformed EEG delta power density (logDelta) and \%HF during the 15- and 45- min naps. The EEG delta power density during the 15-min nap was comparable to that observed during the first 15 minute of 45-min nap. Similar temporal patterns were observed for the logDelta and the \%HF, with an increase in the middle of the 45-min nap.

The results of correlation analysis are summarized in Table 1. The $\log$ SWE was positively correlated with the P300 latency $30 \mathrm{~min}$ after the naps $(\mathrm{r}=0.476, \mathrm{p}<0.05)$, but not significantly with the P300 latency $3 \mathrm{hr}$ after the naps (Fig. 2). The averaged $\log S W E$ during the 45 -min nap was greater than that during the 15 -min nap ( $3.31 \pm 0.41$ vs. $2.61 \pm 0.59$, $\mathrm{F}=8.90, \mathrm{p}<0.01$ ). Figure 3 and Table 1 show that the $\% \mathrm{HF}$ during the naps was negatively correlated with the P300 latency $3 \mathrm{hr}$ after the naps $(\mathrm{r}=-0.519, \mathrm{p}<0.05)$ and was positively correlated with the $\mathrm{P} 300$ amplitude $30 \mathrm{~min}$ after

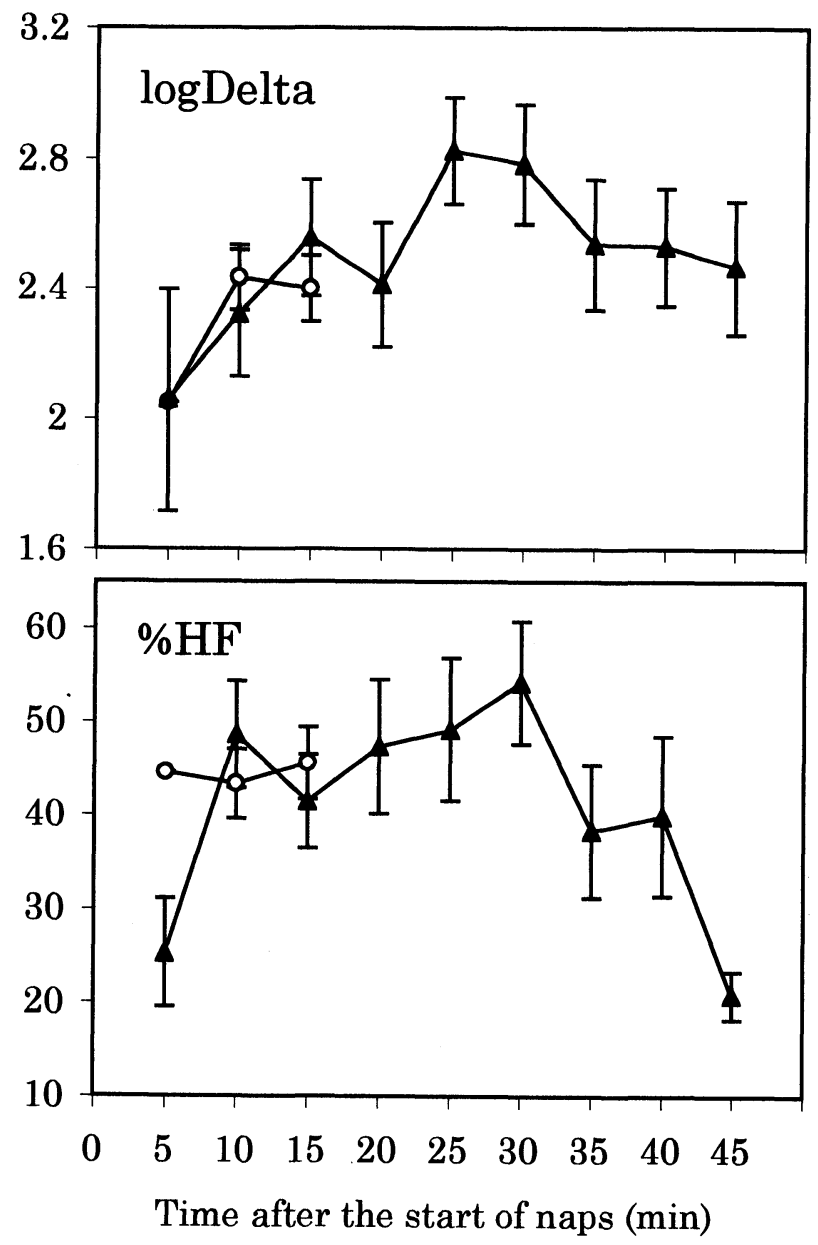

Fig. 1. Time courses of log-transformed EEG delta power density (logDelta) and \%HF during the 15- $(O)$ and 45-min ( $\triangle$ ) naps. Values are means \pm SE. The first values of the $\log$ Delta and $\% \mathrm{HF}$ during the 15-min nap were derived from data of one subject. 

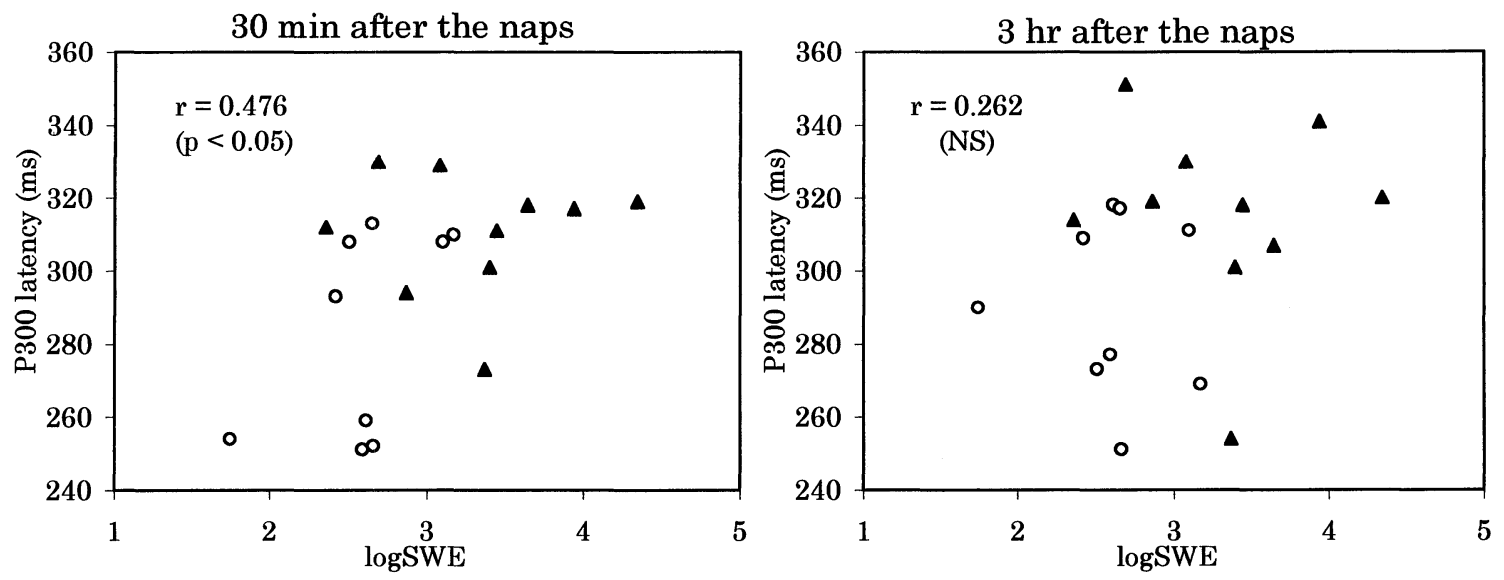

Fig. 2. Relationship between log-transformed slow-wave energy (logSWE) and P300 latency 30 min after and 3 hr after the 15- $(\bigcirc)$ and 45-min ( $\triangle$ ) naps.

The P300 latency $30 \mathrm{~min}$ after the nap was significantly shorter under the 15 -min nap condition $(284.1 \pm 8.5 \mathrm{SE} \mathrm{msec})$ than under the 45-min nap condition ( $310.4 \pm 5.4 \mathrm{msec}, \mathrm{p}<0.05)$. The P300 latency $3 \mathrm{hr}$ after the nap was not significantly different between the 15 - and 45 -min nap conditions ( $294.5 \pm 8.3$ vs. $315.5 \pm 8.3 \mathrm{msec})$.
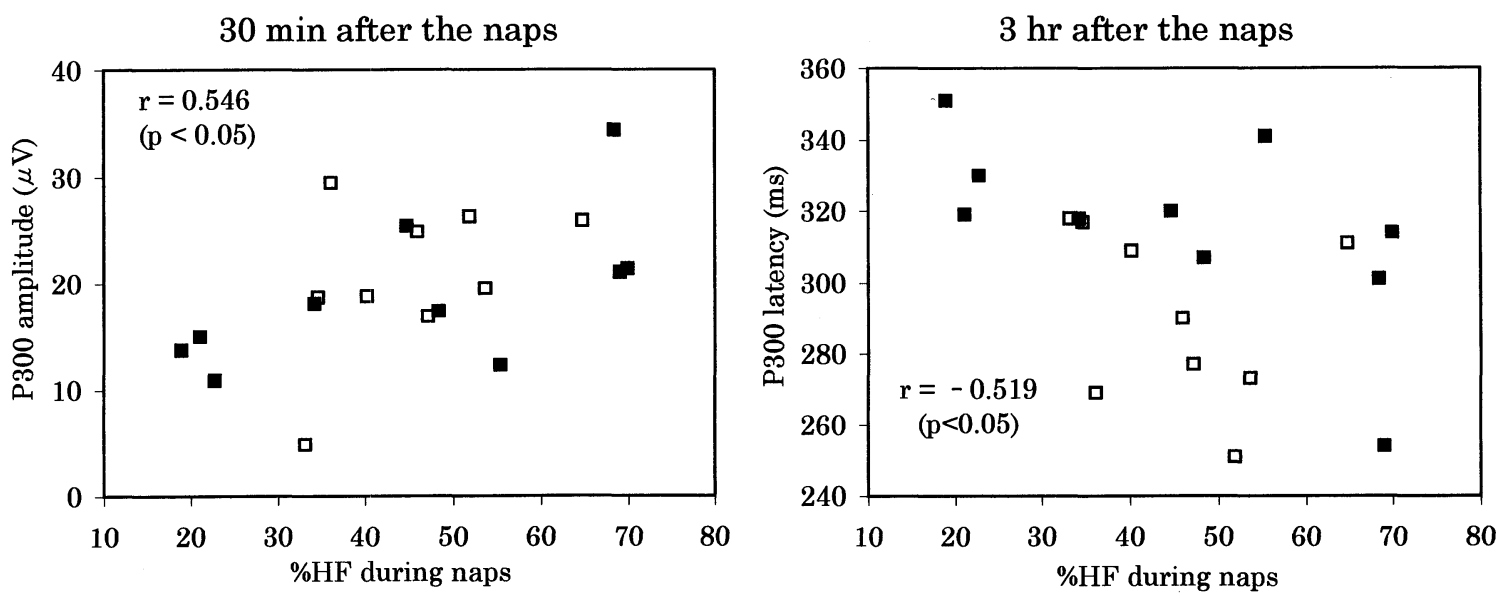

Fig. 3. Relationship between \%HF during the naps and P300 amplitude 30 min after the 15- ( $\square$ ) and 45-min ( $\square$ ) naps or P300 latency $3 \mathrm{hr}$ after the naps.

The \%HF during the naps was comparable between the 15 - and 45 -min naps ( $45.3 \pm 3.5$ vs. $45.3 \pm 6.4 \%$ ). The P300 amplitude 30 min after the naps was not significantly different between the 15- and 45-min nap conditions (20.9 $\pm 2.2 \mathrm{vs.} 18.9 \pm 2.2 \mu \mathrm{V})$.

the naps $(\mathrm{r}=0.546, \mathrm{p}<0.05)$. None of the correlations were significant between the logSWE, \%HF, \%LF, or meanRR during the naps and the subjective sleepiness and error rates after the naps (Table 1).

\section{Discussion}

The result of this study indicates that the P300 latency 30 min after the naps was prolonged with an increase in SWE. The SWE, an integrated EEG delta power density over a
NREM sleep episode, is shown to be a sensitive indicator for the amount of deep NREM sleep rather than the visually scored SWS ${ }^{15)}$. The SWE enabled us to quantify the amount of deep NREM sleep during the 15-min nap without the SWS. The P300 latency is reported to correspond to the stimulus evaluation time ${ }^{24)}$; its prolongation is suggested to reflect a decrement in alertness ${ }^{13)}$. Hence, the present result is interpreted as post-nap slowing of the stimulus evaluation as a function of the increased amount of deep NREM sleep during the naps. This finding supports our assumption that 
Table 1. Spearman rank correlation coefficients of the physiological parameters during the naps with the $\mathrm{P300}$ measures, subjective sleepiness, and error rates in the transcription task after the naps

\begin{tabular}{lcccr}
\hline & P300 latency & P300 amplitude & sleepiness & error rates \\
\hline--30 min after the naps -- & & & \\
logSWE & $0.476^{*}$ & 0.039 & 0.237 & 0.214 \\
\%HF & -0.233 & $0.546^{*}$ & -0.002 & -0.082 \\
\%LF & 0.146 & -0.407 & -0.028 & 0.149 \\
meanRR & -0.248 & 0.230 & 0.075 & -0.084 \\
-- 3 hr after the naps --- & & & \\
logSWE & 0.262 & 0.086 & 0.032 & 0.079 \\
\%HF & $-0.519 *$ & 0.393 & -0.026 & -0.142 \\
\%LF & 0.306 & -0.172 & -0.051 & 0.112 \\
meanRR & -0.257 & 0.253 & -0.207 & -0.349 \\
\hline
\end{tabular}

$* \mathrm{p}<0.05$.

sleep inertia may have caused no significant decrease in P300 latency 30 min after the 45-min daytime nap.

No significant association of the SWE with the P300 latency $3 \mathrm{hr}$ after the naps shows a typical characteristic of sleep inertia that its effect appears only immediately after awakening from sleep ${ }^{8}$. The design of our experiment, however, can not determine precise duration of sleep inertia in a period between $30 \mathrm{~min}$ and $3 \mathrm{hr}$ after the naps. To achieve this, repeated testing with a short interval is required following awakening from naps ${ }^{20)}$. Another design is to examine data measured continuously (e.g., for $30 \mathrm{~min}$ ) per subdivided units of time (e.g., $3 \mathrm{~min}$ ), as employed in a recent study ${ }^{21}$.

In the present study, the correlation between SWE and error rates in the transcription task failed to be significant. If sleep inertia continued about $30 \mathrm{~min}$ as reported previously ${ }^{7,21)}$, we might have missed any sleep inertia effect on the error rates during the 90 -min task period starting at $30 \mathrm{~min}$ after the naps. Previous studies, however, demonstrated that accuracy measures of the cognitive tasks may be less affected by sleep inertia than speed ones ${ }^{19-21)}$. Salame et al $^{21)}$ found no decrement in error rates for spatial memory and logical reasoning tasks immediately after a 1$h$ nap. From this result, they insisted that maintenance of the task accuracy was essential in real work settings, even if the response speed was to some extent delayed. It may be thus reasonable to reconsider negative views of napping that, for example, it is better not to take a nap during a work break because subsequent job performance may be impaired by sleep inertia. Also, it is important to investigate measures to cope with sleep inertia as an inevitable consequence of napping. Environmental stimuli including noise, light, and exercise have been supposed to help to abolish sleep inertia rapidly $^{8,21)}$.

The present result indicates that the \%HF during the naps was negatively associated with the P300 latency $3 \mathrm{hr}$ after the naps. An enhanced parasympathetic activity has been shown to be important for cardiovascular health ${ }^{25,26)}$. The result of Kageyama et al. ${ }^{27)}$ demonstrated that the parasympathetic activity as measured by the HF component was suppressed by work-related fatigue due to long overtime work and commuting. As such, it seems possible that the parasympathetic activity may be enhanced by interventions to recover from fatigue. In this regard, sleeping/napping is advantageous ${ }^{17,18}$. The present data therefore suggests that the parasympathetic predominance produced by napping may be effective for subsequent alertness as assessed by the shortened P300 latency $3 \mathrm{hr}$ after the naps.

The positive association of the $\mathrm{P} 300$ amplitude $30 \mathrm{~min}$ after the naps with the \%HF during the naps was observed in the present study. This is consistent with a previous finding of a very slight increase in P300 amplitude after a 1-hr nap ${ }^{28)}$. The P300 amplitude has been shown to reflect allocation of attentional resources ${ }^{29)}$ and to be attenuated as a result of sleep deprivation ${ }^{30)}$ and long hours of work ${ }^{31)}$. It can be thus surmised that the parasympathetic predominance occurring during the naps may result in increased allocation of attentional resources. The findings of this study show that the parasympathetic activity during the naps may affect the post-nap latency and amplitude of P300 ERP in a different way. Factors based upon these relationships remain unclear, and require further investigation. 
In conclusion, an increase in EEG delta power density during naps produces a prolonged P300 latency immediately after the naps. This demonstrates the effect of sleep inertia. An increased HF component during the naps shortens the P300 latency a few hours after the naps, suggesting an improvement in subsequent alertness associated with parasympathetic predominance during the naps.

\section{Acknowledgments}

The authors are very grateful to Professor Dr. Shosuke Suzuki, Department of Public Health, Gunma University School of Medicine, for his guidance and valuable advice throughout the study. The authors also are grateful to Dr. Roger R. Rosa, the National Institute for Occupational Safety and Health, USA, for his helpful comments during the preparation of this manuscript.

\section{References}

1) Taub JM, Tanguay PE, Rosa RR (1977) Effects of afternoon naps on physiological variables, performance and self-reported activation. Biol Psychol 5, 191-210.

2) Dinges DF (1992) Adult napping and its effects on ability to function. In: Why we nap. ed. by Stampi C, 118-34, Birkhauser, Boston.

3) Gillberg M, Kecklund G, Axelsson J, Akerstedt T (1996) The effects of a short daytime nap after restricted night sleep. Sleep 19, 570-5.

4) Horne JA, Reyner LA (1996) Counteracting driver sleepiness: effects of napping, caffeine, and placebo. Psychophysiol 33, 306-9.

5) Reyner LA, Horne JA (1997) Suppression of sleepiness in drivers: combination of caffeine with a short nap. Psychophysiol 34, 721-5.

6) Takahashi M, Fukuda H, Arito H (1998) Brief naps during post-lunch rest: effects on alertness, performance, and autonomic balance. Eur J Appl Physiol 78, 93-8.

7) Naitoh $P$ (1992) Minimal sleep to maintain performance: the search for sleep quantum in sustained operations. In: Why we nap. ed. by Stampi C, 199-216, Birkhauser, Boston.

8) Muzet A, Nicolas A, Tassi P, Dewasmes G, Bonneau A (1995) Implementation of napping in industry and the problem of sleep inertia. J Sleep Res 4 (Suppl. 2), 679.

9) Dinges DF, Orne MT, Orne EC (1985) Assessing performance upon abrupt awakening from naps during quasi-continuous operations. Behav Res Meth Instr Comp 17, 37-45.

10) Rosa RR, Bonnet MH, Warm JS (1983) Recovery of performance during sleep following sleep deprivation. Psychophysiol 20, 152-9.

11) Rosa RR, Bonnet MH (1985) Sleep stages, auditory arousal threshold, and body temperature as predictors of behavior upon awakening. Int J Neurosci 27, 7383.

12) Picton TW (1992) The $P 300$ wave of the human eventrelated potentials. J Clin Neurophysiol 9, 456-79.

13) Polich J, Kok A (1995) Cognitive and biological determinants of P300: an integrative review. Biol Psychol 41, 103-46.

14) Borbely AA, Baumann F, Brandeis D, Strauch I, Lehmann D (1981) Sleep deprivation: effect on sleep stages and EEG power density in man. Electroencephalogr Clin Neurophysiol 51, 483-95.

15) Akerstedt $T$, Gillberg $M$ (1986) Sleep duration and the power spectral density of the EEG. Electroencephalogr Clin Neurophysiol 64, 119-22.

16) Dijk DJ, Brunner DP, Borbely AA (1990) Time course of EEG power density during long sleep in humans. Am J Physiol 258, R650-61.

17) Van de Borne $P$, Nguyen $H$, Biston $P$, Linkowski $P$, Degaute J (1994) Effects of wake and sleep stages on the 24-h autonomic control of blood pressure and heart rate in recumbent men. Am J Physiol 266, H548-54.

18) Bonnet MH, Arand DL (1997) Heart rate variability: sleep stage, time of night, and arousal influences. Electroencephalogr Clin Neurophysiol 102, 390-6.

19) Naitoh P, Kelly T, Babkoff $H$ (1993) Sleep inertia: best time not to wake up? Chronobiol Int 10, 109-18.

20) Achermann P, Werth E, Dijk DJ, Borbely AA (1995) Time course of sleep inertia after nighttime and daytime sleep episodes. Arch Ital Biol 134, 109-19.

21) Salame P, Otzenberger H, Ehrhart J, Dewasmes G, Nicolas A, Tassi P, Libert JP, Muzet A (1995) Effects of sleep inertia on cognitive performance following a 1-hour nap. Work Stress 9, 528-39.

22) Monk TH (1987) Subjective ratings of sleepinessthe underlying circadian mechanisms. Sleep 10, 34353.

23) Rechtschaffen A, Kales A (1968) A manual of standardized terminology, techniques and scoring system for sleep stages of human subjects. US Department of Health, Education and Welfare, Public Health Service, Bethesda. 
24) McCarthy G, Donchin E (1981) A metric for thought: a comparison of $\mathrm{P} 300$ latency and reaction time. Science 211, 77-80.

25) Malliani A, Pagani M, Lombardi F, Cerutti S (1991) Cardiovascular neural regulation explored in the frequency domain. Circulation 84, 482-92.

26) Kristal-Boneh E, Raifel M, Froom P, Ribak J (1995) Heart rate variability in health and disease. Scand $\mathbf{J}$ Work Environ Health 21, 85-95.

27) Kageyama T, Nishikido N, Kobayashi T, Kurokawa $Y$, Kabuto M (1997) Commuting, overtime, and cardiac autonomic activity in Tokyo. Lancet 350, 639.

28) Wesensten NJ, Badia P, Harsh J (1990) Time of day, repeated testing, and interblock interval effects on P300 amplitude. Physiol Behav 47, 653-8.

29) Wickens C, Kramer A, Vanasse L, Donchin E (1983) Performance of concurrent tasks: a psychophysiological analysis of the reciprocity of information-processing resources. Science 221, 1080-2.

30) Morris AM, So Y, Lee KA, Lash AA, Becker CE (1992) The P300 event-related potential. The effects of sleep deprivation. J Occup Med 34, 1143-52.

31) Emdad R, Belkic K, Theorell T, Wennberg A, Hagman M, Johansson L, Savic C, Cizinsky S (1996) Electrocortical responses to ecologically relevant visual stimuli among professional drivers with and without cardiovascular disease. Integr Physiol Behav Sci 31, 96-111. 